\title{
Entropic segregation of short polymers to the surface of a polydisperse melt
}

\author{
P. Mahmoudi ${ }^{1}$ and M.W. Matsen ${ }^{1,2,3, a}$ \\ 1 Department of Chemical Engineering, University of Waterloo, Waterloo, Ontario, Canada \\ 2 Department of Physics \& Astronomy, University of Waterloo, Waterloo, Ontario, Canada \\ 3 Waterloo Institute for Nanotechnology, University of Waterloo, Waterloo, Ontario, Canada
}

Received 3 August 2017 and Received in final form 18 September 2017

Published online: 6 October 2017

(c) The Author(s) 2017. This article is published with open access at Springerlink.com

\begin{abstract}
Chain ends are known to have an entropic preference for the surface of a polymer melt, which in turn is expected to cause the short chains of a polydisperse melt to segregate to the surface. Here, we examine this entropic segregation for a bidisperse melt of short and long polymers, using self-consistent field theory (SCFT). The individual polymers are modeled by discrete monomers connected by freely-jointed bonds of statistical length $a$, and the field is adjusted so as to produce a specified surface profile of width $\xi$. Semi-analytical expressions for the excess concentration of short polymers, $\delta \phi_{s}(z)$, the integrated excess, $\theta_{s}$, and the entropic effect on the surface tension, $\gamma_{\mathrm{en}}$, are derived and tested against the numerical SCFT. The expressions exhibit universal dependences on the molecular-weight distribution with model-dependent coefficients. In general, the coefficients have to be evaluated numerically, but they can be approximated analytically once $\xi \gtrsim a$. We illustrate how this can be used to derive a simple expression for the interfacial tension between immiscible A- and B-type polydisperse homopolymers.
\end{abstract}

\section{Introduction}

According to a clever argument by Silberberg [1], the surface of a polymer melt can be treated as a reflecting boundary. However, there are various effects that are neglected by this simplified boundary condition. For instance, simulations [2-8] have found an entropic enrichment of chain ends at the surface, whereas a reflecting boundary gives a uniform bulk concentration right up to the surface. Furthermore, experiments [9-11] have observed a molecular-weight dependence in the surface tension, whereas the Silberberg argument predicts none. Naturally, these effects are due to violations in the assumptions used by Silberberg, namely the presumptions of a step-like concentration profile and the absence of an energy penalty for folding polymer chains.

Wu et al. [12] developed the first theoretical treatment for the chain-end segregation and the molecular-weight dependence of the surface tension by applying self-consistent field theory (SCFT) to a compressible polymer melt of Gaussian chains. In that case, the violation to the Silberberg treatment resulted from the finite width of the concentration profile, $\xi$, which increases as the polymer becomes more compressible. More recently, we [13] observed the same behaviors for the SCFT of an incompressible melt with a sharp step profile, where this time the chains

\footnotetext{
a e-mail: mwmatsen@uwaterloo.ca
}

were represented by $N$ beads of volume $\rho_{0}^{-1}$ connected by freely-jointed bonds of statistical length $a$. Here, the violation occurred because reflecting portions of a discrete chain about a plane alters the bonds that initially straddle the plane [14].

In the case of polydisperse melts, the chain-end segregation causes an enrichment of short chains near the surface, since they have more ends per unit volume. This has a wide range of implications in regards to surface tension $[15,16]$, wall slip $[17,18]$, the glass transition of thin films $[19,20]$, and the effective force between polymer surfaces [21], to name a few. The phenomenon also has similar implications for mixtures of chemically identical molecules of different architecture [22-26].

The surface enrichment of short polymers was first demonstrated by Hariharan et al. [27] by applying SCFT to a lattice model of an incompressible bidisperse melt of short and long chains. They investigated both the amplitude and the range of the enrichment. Interestingly, they found that the effect on the surface tension, $\gamma_{\mathrm{en}}$, was consistent with the simple expression

$$
\frac{\gamma_{\mathrm{en}}}{a \rho_{0} k_{B} T}=0.1842-\frac{0.1934}{N_{n}}
$$

where

$$
N_{n}=\left(\sum_{N} \frac{\bar{\phi}_{N}}{N}\right)^{-1}
$$


is the number-average polymerization and $\bar{\phi}_{N}$ is the volume fraction of chains with polymerization $N$. (For their lattice model, $a$ equals the lattice spacing and $\rho_{0}=a^{-3}$.) Van der Gucht et al. [28] extended the lattice SCFT calculations to polydisperse melts with multiple chain lengths. They found that the integrated excess of chains of polymerization $N$ also obeyed a strikingly simple expression

$$
\frac{\theta_{N}}{a \rho_{0}}=0.195 \bar{\phi}_{N}\left(1-\frac{N}{N_{w}}\right),
$$

where

$$
N_{w}=\sum_{N} N \bar{\phi}_{N}
$$

is the weight-average polymerization.

Minnikanti et al. [29] later derived analogous expressions for the enthalpic attraction of chain ends to a surface. They modeled the polymers as Gaussian chains and treated the surface as a reflecting boundary with a Diracdelta potential of strength $U_{e}$ attracting the chain ends. The model was then solved in the mean-field approximation using linear response theory. In their case, the 0.1934 in eq. (1) and the 0.195 in eq. (3) were both replaced by $-2 U_{e} / a$. By considering the limit of infinitely long polymers and assuming an equivalence between enthalpic and entropic segregation [22], they derived an effective potential of $-2 U_{e} / a=0.1951$ for the entropic attraction [30], which nicely agrees with the lattice SCFT calculations of Hariharan et al. and Van der Gucht et al.

Here, we construct an off-lattice SCFT for a polydisperse melt of bead-spring chains with an arbitrary surface profile to examine the entropic enrichment of short chains at the surface. This version of SCFT retains effects due to the discreteness of polymer chains not captured by the Gaussian-chain model, while doing away with the artifacts of a lattice. We also approximate the SCFT by a semianalytical theory, which leads to expressions for $\theta_{N}$ and $\gamma_{\text {en }}$ analogous to those in eqs. (1) and (3), but without having to assume an equivalence with enthaplic attractions. The accuracy of the semi-analytical theory is thoroughly tested against the numerical SCFT for the special case of bidisperse melts, and the relative importance of the surface width and the chain discreteness are assessed.

\section{Numerical SCFT}

This section describes our numerical self-consistent field theory (SCFT) for a polydisperse melt with a surface of area $\mathcal{A}$ located at $z=0$. The individual polymers are modeled by $N$ monomers (or beads), each of volume $\rho_{0}^{-1}$, connected by freely-jointed bonds (or springs). In the case of a polydisperse melt, the dimensionless polymer concentration,

$$
\phi(z)=\sum_{N} \phi_{N}(z)
$$

is a sum of contributions from all the different chain lengths. The bulk concentration of each polymerization $N$ is defined by $\bar{\phi}_{N} \equiv \lim _{z \rightarrow \infty} \phi_{N}(z)$.
We solve this system in the grand-canonical ensemble [31], where the number of chains of polymerization $N$ is controlled by the chemical potential $\mu_{N}$. The nonbonded interactions experienced by a monomer are represented by a field, $w(z)$, which is a function of distance from the surface, $z$. Given this approximation,

$$
\phi_{N}(z)=\frac{z_{N}}{h(z)} \sum_{i=1}^{N} G_{i}(z) G_{N+1-i}(z)
$$

where $z_{N}=\exp \left(\mu_{N} / k_{B} T\right)$ and $h(z) \equiv \exp \left(-w(z) / k_{B} T\right)$. The quantity $G_{i}(z)$ is the partition function for a chain fragment of $i$ monomers with one end fixed at $z$. It is evaluated using the recursive relation

$$
G_{i+1}(z)=h(z) \int g(Z) G_{i}(z-Z) \mathrm{d} Z,
$$

starting from $G_{1}(z)=h(z)$ [14]. The function $g(Z)$ is determined by the bond potential. We will restrict our current study to simple harmonic springs, for which [14]

$$
g(Z)=\left(\frac{3}{2 \pi a^{2}}\right)^{1 / 2} \exp \left(-\frac{3 Z^{2}}{2 a^{2}}\right)
$$

where $a$ is the statistical length of the bond. Since the bonds are freely jointed, the average end-to-end length of an unperturbed polymer is $R_{0}=a(N-1)^{1 / 2} \approx a N^{1 / 2}$. Without loss of generality, we set the field to zero in the bulk, which implies that $G(z) \rightarrow 1$ for large $z$. This, in turn, implies that the fugacity of polymers of polymerization $N$ is

$$
z_{N}=\frac{\bar{\phi}_{N}}{N}
$$

In general, the SCFT needs to be supplemented with a functional, $U[\phi]$, specifying the energy of the non-bonded interactions for a given polymer profile, $\phi(z)$. The field, $w(z)$, is then adjusted to satisfy a self-consistent condition involving the functional derivative of $U[\phi]$, and from that emerges a prediction for the equilibrium profile. However, this is a relatively separate issue from the entropic segregation. In any case, we know from detailed molecular dynamics simulations [7] that the polymer concentration roughly exhibits a sigmoidal profile,

$$
\phi(z)=\frac{1}{2}\left[1+\tanh \left(\frac{2 z}{\xi}\right)\right],
$$

of some width $\xi$. So rather than complicating our calculations with a sufficiently realistic $U[\phi]$, we adjust $w(z)$ to create a sigmoidal profile of some specified width $\xi$, which we treat as a system parameter. The adjustment is done numerically using the Anderson-mixing [32] algorithm described in ref. [33]. Later on, we will discuss an analytical way of determining the equilibrium profile, after we derive the dependence of $\gamma_{\mathrm{en}}$ on $\phi(z)$.

Once the field is determined, we can immediately calculate the excess concentration of chains of polymerization $N$,

$$
\delta \phi_{N}(z)=\phi_{N}(z)-\bar{\phi}_{N} \phi(z)
$$


and from that the integrated excess,

$$
\theta_{N}=\rho_{0} \int \delta \phi_{N}(z) \mathrm{d} z
$$

To obtain the surface tension, we need the grand-canonical free energy

$$
F_{g c}=-k_{B} T \rho_{0} \sum_{N} z_{N} Q_{N}-\rho_{0} \mathcal{A} \int w(z) \phi(z) \mathrm{d} z
$$

where $Q_{N}$ is the single-chain partition function for a polymer of polymerization $N$, which can be evaluated by

$$
Q_{N}=\mathcal{A} \int \frac{G_{i}(z) G_{N+1-i}(z)}{h(z)} \mathrm{d} z
$$

for any value of $i$ from 1 to $N$. Comparing eqs. (6) and (14), it follows that

$$
z_{N} Q_{N}=\frac{\mathcal{A}}{N} \int \phi_{N}(z) \mathrm{d} z
$$

The surface tension, which equals the excess free energy per unit area, can then be expressed as

$$
\gamma_{\mathrm{en}}=-k_{B} T \sum_{N} \frac{\theta_{N}}{N}-\rho_{0} \int w(z) \phi(z) \mathrm{d} z .
$$

\section{Semi-analytical theory}

We now approximate the SCFT with a semi-analytical theory derived using the approach in refs. [12] and [13]. The first step is to evaluate the field $w_{\infty}(z)$ and partition function $G_{\infty}(z)$ for an infinitely long polymer, which turns out to be a relatively simple numerical calculation [33]. As we have shown previously [33], the excess concentration of finite chains of polymerization $N$ in the field $w_{\infty}(z)$ is

$$
\begin{aligned}
\delta \phi_{N}^{0}(z)= & 4 A \bar{\phi}_{N} \sqrt{\frac{6}{\pi N}} \frac{\phi(z)}{G_{\infty}(z)} \\
& \times\left\{\left[f\left(z / a N^{1 / 2}\right)-1\right] H(z)+G_{\infty}(z)\right\},
\end{aligned}
$$

where $H(z)$ is the Heaviside step function that switches from zero to one at $z=0$,

$$
f(\zeta)=\int_{0}^{1} \frac{1}{2 \sqrt{s}} \exp \left(-\frac{3}{2 s} \zeta^{2}\right) \mathrm{d} s
$$

and

$$
A=\frac{1}{a} \int\left(G_{\infty}(z)-\phi(z)\right) \mathrm{d} z
$$

The coefficient $A$ in eq. (17) depends on the width of the surface, $\xi$, as shown in fig. 1.

Naturally, the total excess concentration, $\sum_{N} \delta \phi_{N}^{0}(z)$, will be non-zero, and thus the polymer concentration will

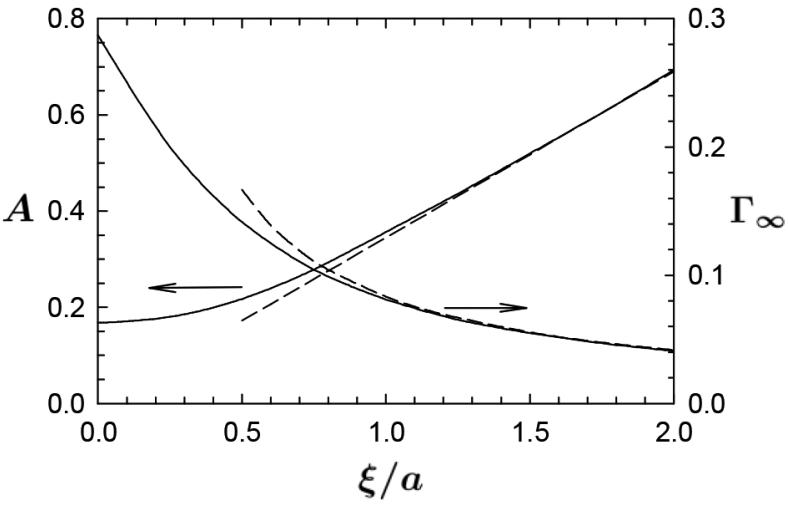

Fig. 1. Numerical values of $A$ and $\Gamma_{\infty}$ evaluated using eqs. (19) and (34), respectively. The dashed curves compare the analytical approximations in eqs. (44) and (45) corresponding to the Gaussian-chain model.

deviate from our target profile in eq. (10). The main source of deviation comes from the long-range behavior,

$$
\delta \phi_{N}^{0}(z) \approx 4 A \bar{\phi}_{N} \sqrt{\frac{6}{\pi N}} f\left(z / a N^{1 / 2}\right),
$$

at $z \gg a$. To restore the polymer profile to eq. (10), we must adjust the field,

$$
w(z)=w_{\infty}(z)+\delta w(z),
$$

by some appropriate amount $\delta w(z)$. For this, we need to know the change in concentration of each molecular weight, $\delta \phi_{N}^{1}(z)$, due to $\delta w(z)$.

For high molecular weights, the correction to the field will be small and slowly varying, which allows us to employ linear response theory while approximating the polymers as Gaussian chains and the surface as a reflecting boundary. The boundary condition is accounted for by treating $\delta \phi_{N}^{1}(z)$ and $\delta w(z)$ as even functions of $z$. The linear response theory for Gaussian chains [31] states that the Fourier transforms ${ }^{1}$,

$$
\widehat{g}\left(k_{z}\right) \equiv \int_{-\infty}^{\infty} g(z) e^{-i k_{z} z} \mathrm{~d} z,
$$

of $\delta \phi_{N}^{1}(z)$ and $\delta w(z)$ are related by

$$
\widehat{\delta \phi_{N}^{1}}\left(k_{z}\right)=-N \bar{\phi}_{N} s_{\phi}\left(x_{N}\right) \frac{\widehat{\delta w}\left(k_{z}\right)}{k_{B} T},
$$

where $s_{\phi}(x)=2\left(e^{-x}+x-1\right) / x^{2}$ is the Debye function and $x_{N} \equiv k_{z}^{2} a^{2} N / 6$. The polymer profile is restored by requiring

$$
\sum_{N}\left[\widehat{\delta \phi_{N}^{0}}\left(k_{z}\right)+\widehat{\delta \phi_{N}^{1}}\left(k_{z}\right)\right]=0
$$

The Fourier transform of the approximation for $\delta \phi_{N}^{0}(z)$ in eq. (20) is given by

$$
\widehat{\delta \phi_{N}^{0}}\left(k_{z}\right)=4 A a \bar{\phi}_{N} s_{e}\left(x_{N}\right)
$$

${ }^{1}$ Here we uses a different normalization of the Fourier transforms than in our previous work $[13,33]$. 
where $s_{e}(x)=\left(1-e^{-x}\right) / x$ is a Debye-like function. It then follows from eqs. (23), (24), and (25) that

$$
\frac{\widehat{\delta w}\left(k_{z}\right)}{k_{B} T}=4 A a \frac{\sum_{N} \bar{\phi}_{N} s_{e}\left(x_{N}\right)}{\sum_{N} N \bar{\phi}_{N} s_{\phi}\left(x_{N}\right)} .
$$

The $N$ in the denominator confirms our claim that $\delta w(z)$ becomes smaller in the large-chain limit, which increases the accuracy of the linear response theory.

Now that $\widehat{\delta w}\left(k_{z}\right)$ is known, $\widehat{\delta \phi}_{N}^{1}\left(k_{z}\right)$ can be evaluated using eq. (23) and then $\delta \phi_{N}^{1}(z)$ can be calculated by performing an inverse Fourier transform,

$$
g(z) \equiv \frac{1}{2 \pi} \int_{-\infty}^{\infty} \widehat{g}\left(k_{z}\right) e^{i k_{z} z} \mathrm{~d} k_{z} .
$$

This provides us now with the corrected excess concentration

$$
\delta \phi_{N}(z)=\delta \phi_{N}^{0}(z)+\delta \phi_{N}^{1}(z) .
$$

Using the fact

$$
\int_{0}^{\infty} g(z) \mathrm{d} z=\frac{1}{2} \widehat{g}(0)
$$

it follows that

$$
\theta_{N} \approx \frac{\rho_{0}}{2}\left[\widehat{\delta \phi_{N}^{0}}(0)+\widehat{\delta \phi_{N}^{1}}(0)\right]
$$

and

$$
\begin{aligned}
\gamma_{\text {en }} \approx & -k_{B} T \sum_{N} \frac{\theta_{N}}{N}-\rho_{0} \int w_{\infty}(z) \phi(z) \mathrm{d} z \\
& -\frac{\rho_{0}}{2} \widehat{\delta w}(0) .
\end{aligned}
$$

Noting that $s_{\phi}(0)=s_{e}(0)=1$, eq. (30) simplifies to

$$
\frac{\theta_{N}}{a \rho_{0}} \approx 2 A \bar{\phi}_{N}\left(1-\frac{N}{N_{w}}\right) \text {. }
$$

Combining this with the fact that $\phi(z)=$ $G_{\infty}^{2}(z) / h_{\infty}(z)[33]$ reduces eq. (31) to

$$
\frac{\gamma_{\mathrm{en}}}{a \rho_{0} k_{B} T} \approx \Gamma_{\infty}-\frac{2 A}{N_{n}} .
$$

The coefficient,

$$
\Gamma_{\infty}=\frac{1}{a} \int \phi(z) \ln \left[\frac{G_{\infty}^{2}(z)}{\phi(z)}\right] \mathrm{d} z,
$$

depends on the width of the surface, $\xi$, as shown in fig. 1.

\section{Results}

To make our study more manageable, we consider the special case of a bidisperse melt of short and long polymers of polymerizations $N_{s}$ and $N_{l}=\alpha N_{s}$ and bulk compositions $\bar{\phi}_{s}$ and $\bar{\phi}_{l}=1-\bar{\phi}_{s}$, respectively. This reduces the number

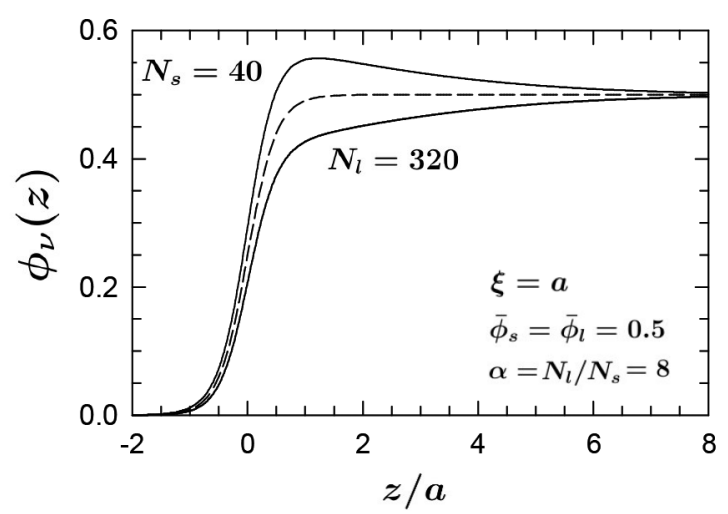

Fig. 2. Polymer concentrations, $\phi_{\nu}(z)(\nu=s$ or $l)$, in a $50: 50$ mixture of $N_{s}=40$ and $N_{l}=320$ polymers, calculated for a surface profile, $\phi(z)$, of width $\xi=a$. The dashed curve denotes the reference concentration, $\phi(z) / 2$, corresponding to zero surface segregation.

of relevant quantities to three: the concentration of short polymers, $\phi_{s}(z)$, the integrated excess of short polymers, $\theta_{s}$, and the surface tension, $\gamma_{\mathrm{en}}$. It also limits the number of independent system parameters to four: the surface width, $\xi$, the ratio of molecular weights, $\alpha=N_{l} / N_{s}$, the bulk composition, $\bar{\phi}_{s}$, and the polymerization of the short polymers, $N_{s}$. The SCFT predictions for the three quantities will be examined with respect to each of these four parameters. While doing so, the semi-analytical predictions will be tested against the SCFT results.

An example of the general behavior is illustrated in fig. 2 for $\xi=a, \alpha=8$, and $\bar{\phi}_{s}=0.5$. The solid curves show the concentration profiles of the short and long polymers, $\phi_{s}(z)$ and $\phi_{l}(z)$, respectively, while the dashed curve denotes the reference concentration corresponding to zero excess, which equals $\phi(z) / 2$ for both components of a 50:50 mixture. The total excess of short molecules, $\theta_{s}$, is obtained by integrating the difference between $\phi_{s}(z)$ and the reference. Naturally, this excess is balanced by a depletion of the long polymers (i.e., $\theta_{l}=-\theta_{s}$ ).

In the case of bidisperse melts, the semi-analytical theory predicts the excess concentration of short chains to be

$$
\delta \phi_{s}(z) \approx \begin{cases}A N_{s}^{-1 / 2} C(0) \phi(z), & \text { for } z<0 \\ A N_{s}^{-1 / 2} C\left(z / a N_{s}^{1 / 2}\right), & \text { for } z>0\end{cases}
$$

where

$$
C(\zeta)=4 \bar{\phi}_{s}\left(\sqrt{\frac{6}{\pi}} f(\zeta)-c(\zeta)\right)
$$

The function $f(\zeta)$ is defined in eq. (18) and the function $c(\zeta)$ is obtained from an inverse Fourier transform of

$$
\hat{c}\left(k_{\zeta}\right)=\frac{s_{\phi}(x)\left[s_{e}(x)+\beta s_{e}(\alpha x)\right]}{s_{\phi}(x)+\alpha \beta s_{\phi}(\alpha x)},
$$

where $x=k_{\zeta}^{2} / 6$ and $\beta=\left(1-\bar{\phi}_{s}\right) / \bar{\phi}_{s}$. Note that the large$\alpha$ limit of $\hat{c}\left(k_{\zeta}\right)$ is obtained by setting $s_{e}(\alpha x)=0$ and $\alpha s_{\phi}(\alpha x)=2 / x$. 

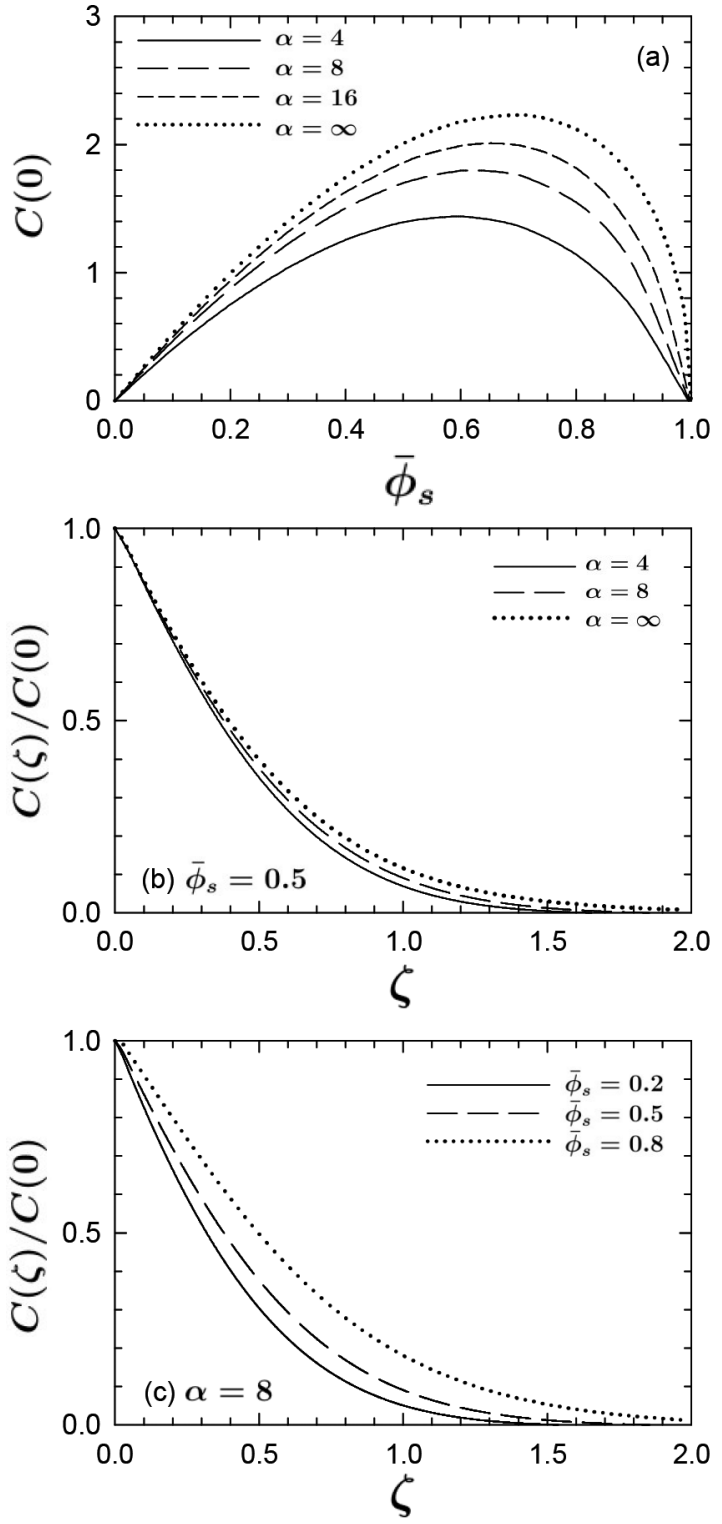

Fig. 3. Dependence of $C(\zeta)$ on $\alpha \equiv N_{l} / N_{s}$ and $\bar{\phi}_{s}=1-\bar{\phi}_{l}$. Plot (a) demonstrates the dependence of its magnitude on the two parameters, while plots (b) and (c) show how its shape changes with $\alpha$ and $\bar{\phi}_{s}$, respectively.

As it turns out, $C(\zeta)$ is a universal function that only depends on the molecular-weight distribution (i.e., the parameters $\alpha$ and $\bar{\phi}_{s}$ ). Figure 3(a) illustrates the dependence of its amplitude, $C(0)$, on the parameters, which qualitatively matches the lattice SCFT calculations of Hariharan et al. (see fig. 1 in ref. [27]). Naturally, $C(0)$ vanishes when $\bar{\phi}_{s}=0, \bar{\phi}_{s}=1$, or $\alpha=1$, since these limits correspond to a monodisperse melt. Figures $3(\mathrm{~b})$ and (c) show that $C(\zeta)$ has an exponential-like decay, which is relatively independent of the parameters. (Note that the lower plot closely resembles fig. 3 of Hariharan et al.) In all cases, the decay length is of order one, which implies that the range of the excess concentration scales with the size of the smaller polymer, $\zeta=a N_{s}^{1 / 2}$, as opposed to the larger polymer, $\zeta \alpha^{1 / 2}=a N_{l}^{1 / 2}$.
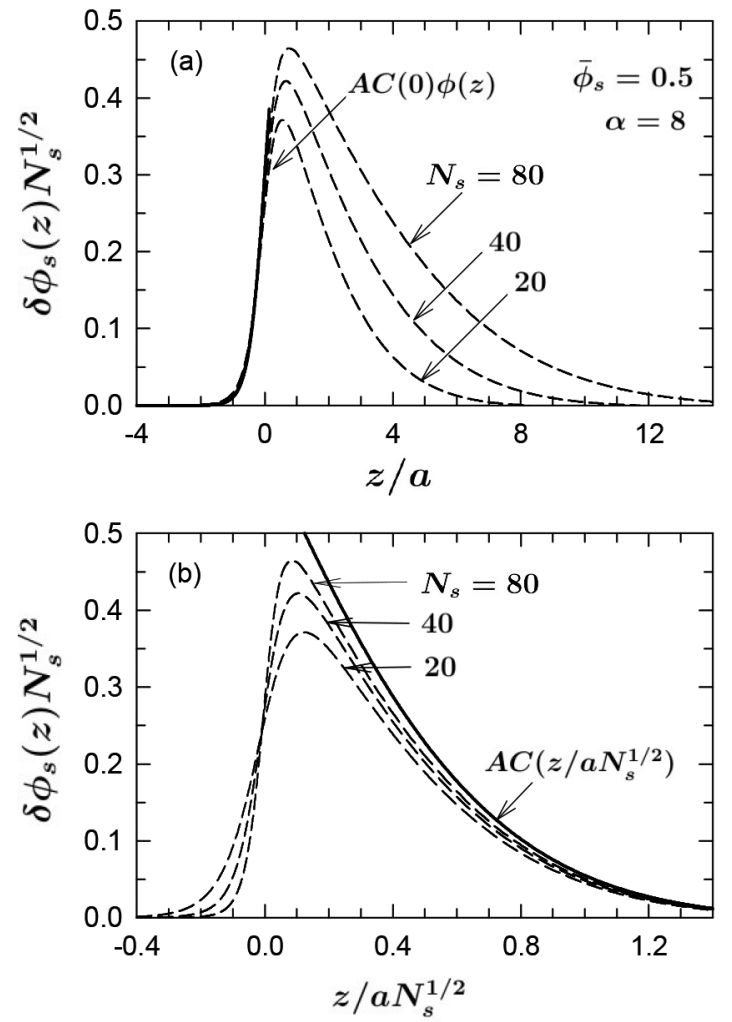

Fig. 4. Excess concentration of short polymers, $\delta \phi_{s}(z) \equiv$ $\phi_{s}(z)-\bar{\phi}_{s} \phi(z)$, plotted on the (a) monomer and (b) molecular length scales for $\alpha=8$ and $\bar{\phi}_{s}=0.5$. Dashed curves denote SCFT results and solid curves correspond to the approximation in eq. (35).

Figure 4 plots SCFT results for the excess concentration of short polymers, $\delta \phi_{s}(z)$, on the (a) monomer and (b) molecular length scales, calculated at $\alpha=8$ and $\bar{\phi}_{s}=0.5$ for several different polymerizations, $N_{s}$. Solid curves denote the semi-analytical approximation in eq. (35). The approximation proves to be very accurate for $z<0$, but less so for $z>0$. However, the accuracy does improve as $N_{s}$ increases, as it must since the linear response theory becomes exact in the large-chain limit.

We now turn our attention to the integrated excess, $\theta_{s}$, and the entropic contribution to the surface tension, $\gamma_{\mathrm{en}}$. In SCFT, these quantities are given by

$$
\frac{\theta_{s}}{a \rho_{0}}=\frac{1}{a} \int \delta \phi_{s}(z) \mathrm{d} z
$$

and

$$
\frac{\gamma_{\mathrm{en}}}{a \rho_{0} k_{B} T}=-\frac{\theta_{s}(\alpha-1)}{a \rho_{0} \alpha N_{s}}-\frac{1}{a k_{B} T} \int w(z) \phi(z) \mathrm{d} z .
$$

The corresponding approximations of the semi-analytical theory are

$$
\frac{\theta_{s}}{a \rho_{0}} \approx \frac{2 A(\alpha-1) \bar{\phi}_{s}\left(1-\bar{\phi}_{s}\right)}{\alpha-(\alpha-1) \bar{\phi}_{s}}
$$

and

$$
\frac{\gamma_{\mathrm{en}}}{a \rho_{0} k_{B} T} \approx \Gamma_{\infty}-\frac{2 A\left[(\alpha-1) \bar{\phi}_{s}+1\right]}{\alpha N_{s}} .
$$



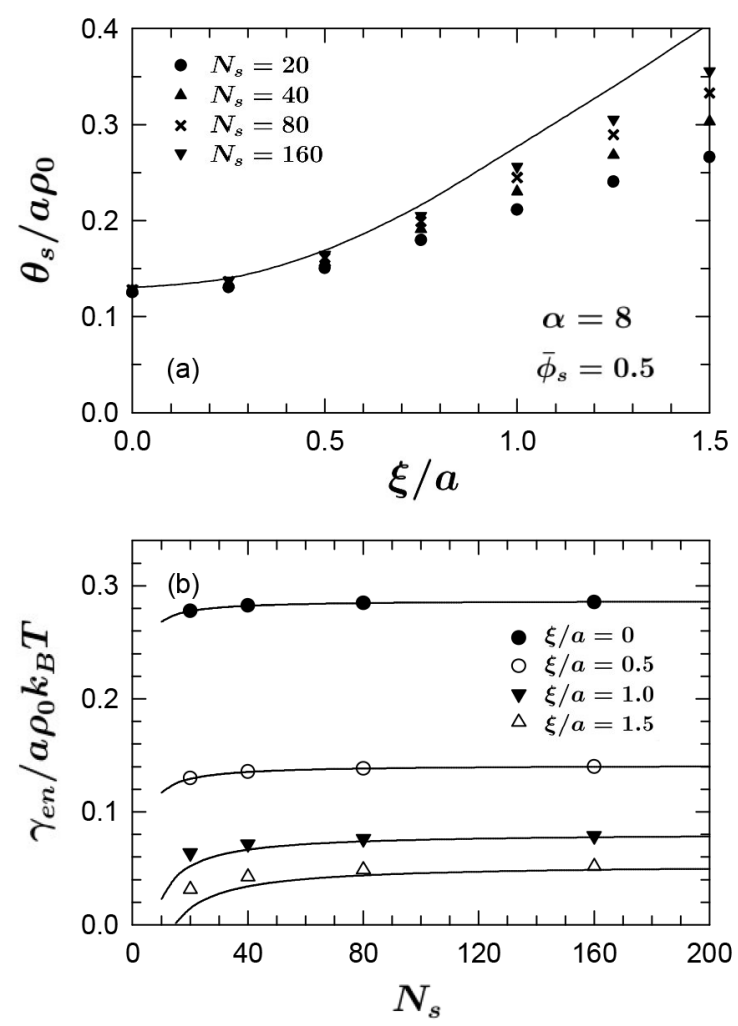

Fig. 5. (a) Surface excess of the short polymer, $\theta_{s}$, as a function of the surface width, $\xi$, calculated for different chain lengths, $N_{s}$. (b) Entropic surface tension, $\gamma_{\mathrm{en}}$, as a function of $N_{s}$ calculated for different values of $\xi$. All results are for fixed values of $\alpha \equiv N_{l} / N_{s}=8$ and $\bar{\phi}_{s}=\bar{\phi}_{l}=0.5$. The SCFT calculations are denoted by symbols and the semi-analytical approximations, eqs. (40) and (41), are plotted with continuous curves.

Figure 5 examines the effect of the surface width, $\xi$, on $\theta_{s}$ and $\gamma_{\mathrm{en}}$, for fixed values of $\alpha=8$ and $\bar{\phi}_{s}=0.5$. Symbols denote the full SCFT calculation for different polymerizations, $N_{s}$, and continuous curves compare the semi-analytical predictions, which in this case are controlled solely by the $\xi$-dependences of $A$ and $\Gamma_{\infty}$ in fig. 1 . While the semi-analytical theory proves to be accurate for the step profile, it becomes less so as $\xi$ increases, particularly in regards to $\theta_{s}$. As before, the accuracy improves for larger $N_{s}$. Interestingly, the level of segregation in fig. 5(a) at $\xi=0$, which is attributed solely to the discreteness of the chains, is a significant fraction of that for the finite widths typical of an air/polymer surface (i.e., $\xi \sim a[2,7$, $8]$ ). This suggests that the importance of chain discreteness is comparable to the details of the surface profile [33], but we will demonstrate later that this is not so.

Next we calculate the effect of the relative chain lengths, $\alpha \equiv N_{l} / N_{s}$, on $\theta_{s}$ and $\gamma_{\text {en }}$ in figs. 6(a) and (b), respectively, for fixed $\xi=a$ and $\bar{\phi}_{s}=0.5$. Naturally, $\theta_{s}$ is zero at $\alpha=1$, since this limit reduces the system to a monodisperse melt. As $\alpha$ increases from one, there is a rapid rise in $\theta_{s}$ that then plateaus. The semi-analytical theory predicts a plateau of $2 A a \rho_{0} \bar{\phi}_{s}\left(1-\bar{\phi}_{s}\right)$. The dependence of $\gamma_{\text {en }}$ on $N_{s}$ is shown in fig. 6(b) for several different values of $\alpha$. There is a slightly stronger $N_{s}$-dependence for
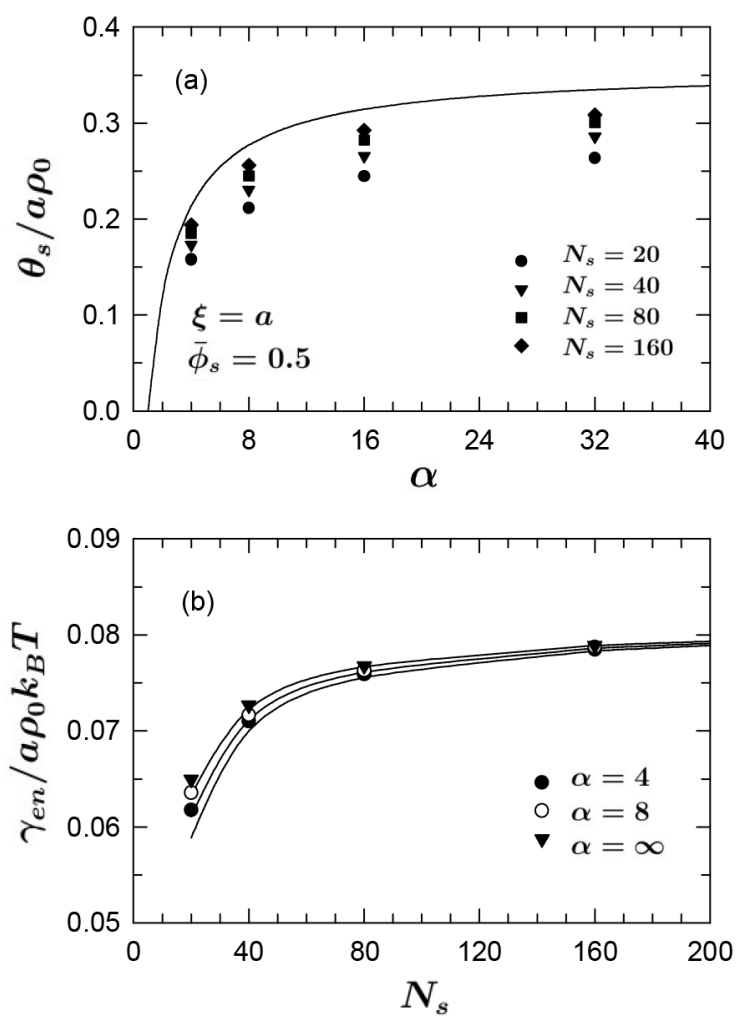

Fig. 6. Analogous plots to those of fig. 5 examining the dependence of $\theta_{s}$ and $\gamma_{\text {en }}$ on $\alpha$ for fixed values of $\xi=a$ and $\bar{\phi}_{s}=\bar{\phi}_{l}=0.5$.
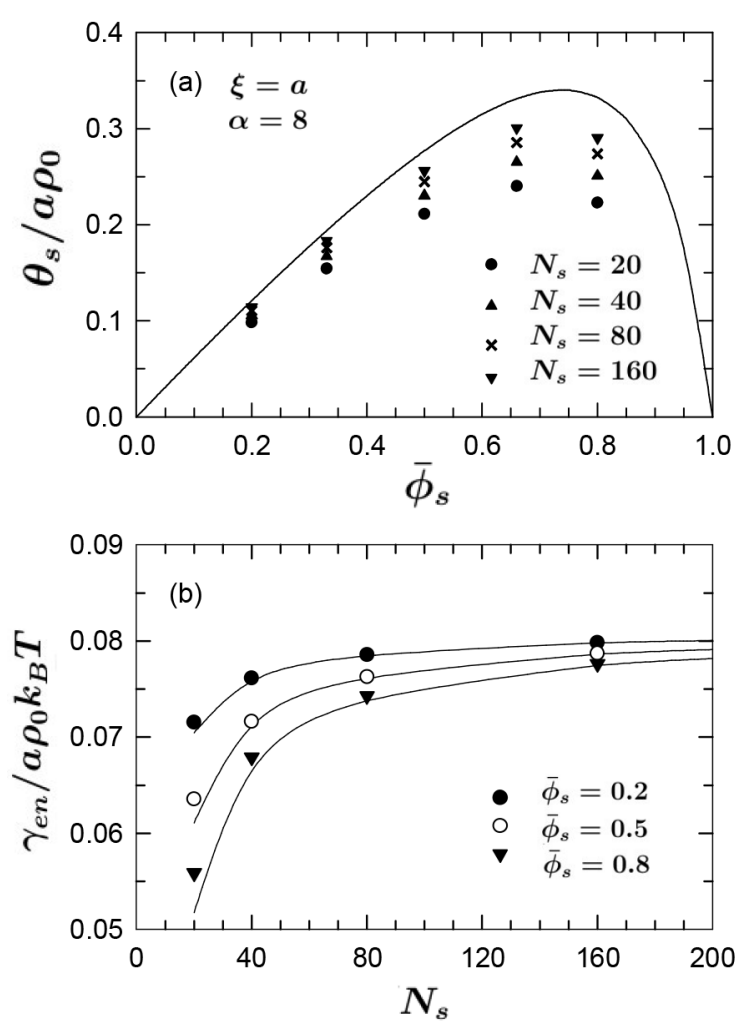

Fig. 7. Analogous plots to those of fig. 5 examining the dependence of $\theta_{s}$ and $\gamma_{\mathrm{en}}$ on $\bar{\phi}_{s}$, for fixed values of $\xi=a$ and $\alpha=8$. 
the smaller values of $\alpha$, because this corresponds to more ends from the long chains. The effect of $\alpha$ is comparatively weak relative to that of $N_{s}$ because the total number of ends is dominated by the short molecules. Once again, the semi-analytical theory is accurate in regards to $\gamma_{\mathrm{en}}$, but not so accurate for $\theta_{s}$.

Lastly, we examine the effect of the blend composition, $\bar{\phi}_{s}$, for fixed $\alpha=8$ and $\xi=a$. Naturally, $\theta_{s}$ vanishes in the limits $\bar{\phi}_{s}=0$ and 1 , since the melt becomes monodisperse. The SCFT results for $\theta_{s}$ in fig. 7(a) (denoted by symbols) peak at an intermediate composition in reasonable agreement with the semi-analytical prediction $\bar{\phi}_{s}^{\max }=1 /\left(1+\alpha^{-1 / 2}\right)$, which in this case corresponds to $\bar{\phi}_{s}^{\max }=0.739$. Figure 7(b) shows the $N_{s}$-dependence of $\gamma_{\text {en }}$ for several different compositions. In this case, there is a sizeable reduction in $\gamma_{\text {en }}$ for larger $\bar{\phi}_{s}$, simply because this greatly increases the number of chain ends. As in all our other examples, the semi-analytical theory is found to be far more accurate in regard to $\gamma_{\mathrm{en}}$ than $\theta_{s}$.

\section{Discussion}

Although the semi-analytical prediction for the excess concentration, $\delta \phi_{N}(z)$, and thus for the integrated excess, $\theta_{N}$, becomes inaccurate at large $\xi$, the theory nevertheless provides an invaluable understanding into the qualitative behavior. Most notably, it reveals that $\delta \phi_{N}(z)$ exhibits a universal shape at $z \gg a$, which depends only on the molecular-weight distribution (i.e., $\left.\bar{\phi}_{N}\right)$, albeit with a nonuniversal amplitude (i.e., $A$ ). Here, we showed that $A$ is dependent on the surface profile, which would be affected, for example, by the compressibility of the melt. We previously illustrated that it also depends on the interaction potential between bonded monomers [13]. In general, $A$ will be affected by all microscopic details, whether it be those of a model or those of an actual system.

In regard to the entropic contribution to surface tension, $\gamma_{\mathrm{en}}$, the semi-analytical theory provides accurate predictions even at large $\xi$. Furthermore, it reproduces the simple inverse dependence on $N_{n}$, first observed in the lattice SCFT of Hariharan et al. [27]. In a previous study for monodisperse melts [33], we showed that the reduction in surface tension obeys

$$
\gamma=\gamma_{\infty}-k_{B} T \Delta \sigma_{e}
$$

where $\Delta \sigma_{e}=2 a \rho_{0} A / N$ is the surface excess of chain ends. Given that the total excess generalizes to

$$
\Delta \sigma_{e}=2 a \rho_{0} A \sum_{N} \frac{\bar{\phi}_{N}}{N}=\frac{2 a \rho_{0} A}{N_{n}}
$$

for polydisperse melts, it immediately follows that eq. (42) is equivalent to eq. (33) and thus also applies to polydisperse melts.

Although our semi-analytical theory is derived for bead-spring chains, it readily extends to the lattice model used by Hariharan et al. and Van der Gucht et al. We simply set $\phi(z)$ to a step function, and replace integrals of $Z$ by sums over the values $-a, 0$, and $a$, for which $g(Z)=1 / 6,2 / 3$, and $1 / 6$, respectively. This gives $2 A=0.1951$ and $\Gamma_{\infty}=0.1842$ for the coefficients in eqs. (1) and (3), in agreement with the alternative derivation by Minnikanti et al. [29]. These values, however, are specific to a simple-cubic lattice with the surface normal to the (001) direction. If one instead considers the (001) surface of an fcc lattice, where $g(Z)=1 / 3$ for the allowed values $Z=-a / \sqrt{2}, 0$ and $\mathrm{a} / \sqrt{2}$, the coefficients become $2 A=0.3789$ and $\Gamma_{\infty}=0.3088$. The fact that the choice of artificial lattice has such a large impact on the entropic segregation implies that off-lattice models are required for accurate predictions.

Our semi-analytical theory for discrete chains can also be extended to continuous Gaussian chains, in which case it becomes fully analytical. When the field acting on the chain is spread evenly along its contour rather than at discrete points, $G_{\infty}(z)$ reduces from $\sqrt{h_{\infty}(z) \phi(z)}$ to $\sqrt{\phi(z)}$. Thus, eq. (19) becomes

$$
A \approx \frac{1}{a} \int(\sqrt{\phi(z)}-\phi(z)) \mathrm{d} z=\frac{\xi \ln 2}{2 a},
$$

which is evaluated and plotted in fig. 1 for the sigmodial profile, eq. (10). Furthermore, the ground-state dominance approximation for Gaussian chains [31] gives

$$
\Gamma_{\infty} \approx \frac{a}{24} \int \frac{[\nabla \phi(z)]^{2}}{\phi(z)} \mathrm{d} z=\frac{a}{12 \xi}
$$

for the surface tension of infinitely long chains, which is also plotted in fig. 1. As mentioned previously, the $\xi$ dependence of $A$ for discrete chains (solid curves) on its own suggests that the effect of the chain discreteness is generally comparable to that of the profile width. However, now that we have evaluated $A$ for continuous chains (dashed curves), it is clear that the effect of chain discreteness quickly vanishes once the profile width reaches values of $\xi \gtrsim a$.

Given this justification for continuous Gaussian chains, the combination of eqs. (33), (44), and (45) should provide an accurate analytical expression for $\gamma_{\mathrm{en}}$ in terms of $\phi(z)$. This, in turn, allows for a straightforward calculation of the equilibrium surface profile, when provided with an appropriate energy functional, $U[\phi]$, for the molecular interactions. One simply solves the Euler-Lagrange equation that minimizes the total surface tension, $\gamma=U[\phi] / \mathcal{A}+\gamma_{\mathrm{en}}$ under the constraint $\int \phi(z) \mathrm{d} z=$ const. We could, for instance, reproduce the surface profile of $\mathrm{Wu}$ et al. $[12,22]$ by using their

$$
\frac{U[\phi]}{\mathcal{A}}=-\frac{\nu \rho_{0}^{2}}{2} \int \phi^{2}(z) \mathrm{d} z,
$$

where $\nu$ is the excluded-volume parameter controlling the compressibility of the melt. In this case, however, we would also have to include the boundary condition, $\phi(0)=0$, because this simplified $U[\phi]$ does not produce two-phase coexistence between melt and vapor states. As pointed out 


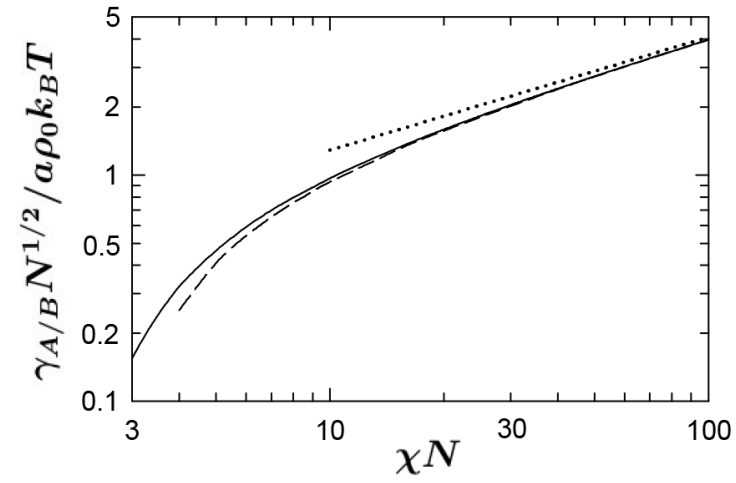

Fig. 8. Interfacial tension between monodisperse A- and Btype homopolymers of polymerization $N$ [31], where $\chi$ is the Flory-Huggins interaction parameter. The dotted line denotes the conventional strong-segregation approximation [37], while the dashed curve shows the improved approximation in eq. (48).

previously [33], this leads to unphysical surface profiles, particularly in the case of discrete chains. Naturally, the problem can be remedied by using a more realistic $U[\phi]$ from, for example, density functional theory [34,35], but that is beyond the scope of this paper.

To illustrate the utility of our analytical expression for $\gamma_{\mathrm{en}}$, consider the A/B interface between immiscible Aand B-type homopolymers of number-average polymerizations, $N_{\mathrm{A}, n}$ and $N_{\mathrm{B}, n}$, respectively. The interfacial tension takes the form $\gamma_{\mathrm{A} / \mathrm{B}}=U[\phi] / \mathcal{A}+\gamma_{\mathrm{A} \text {,en }}+\gamma_{\mathrm{B}, \text { en }}$, where

$$
\frac{U[\phi]}{\rho_{0} k_{B} T \mathcal{A}}=\chi \int \phi(z)[1-\phi(z)] \mathrm{d} z=\frac{\chi \xi}{4}
$$

is the conventional interaction energy controlled by the usual Flory-Huggins parameter, $\chi$. Here, $U[\phi]$ is evaluated for the sigmodial profile, eq. (10), which is the known shape that minimizes the interfacial tension in the infinite molecular-weight limit [31]. Supplementing eq. (33) with eqs. (44) and (45) provides $\gamma_{\mathrm{A} \text {,en }}$ and $\gamma_{\mathrm{B} \text {,en }}$ for the same profile. Then it is just a matter of adding the three contributions of $\gamma_{\mathrm{A} / \mathrm{B}}$ together and minimizing with respect to $\xi$, which results in the simple expression

$$
\frac{\gamma_{\mathrm{A} / \mathrm{B}}}{a \rho_{0} k_{B} T} \approx \sqrt{\frac{\chi}{6}}\left(1-\frac{2 \ln 2}{\chi N_{\mathrm{A}, n}}-\frac{2 \ln 2}{\chi N_{\mathrm{B}, n}}\right) .
$$

Note that this nicely agrees with an earlier derivation by Semenov for monodisperse homopolymers [36]. The approximation is compared in fig. 8 with a numerical SCFT calculation from ref. [31] for monodisperse homopolymers of equal polymerization, $N$. As one can clearly see, eq. (48) provides a marked improvement over the conventional strong-segregation approximation, $\gamma_{\mathrm{A} / \mathrm{B}} \approx a \rho_{0} k_{B} T \sqrt{\chi / 6}$ (dotted curve) [37]. Be aware that our derivation is specific to the case where $\mathrm{A}$ and $\mathrm{B}$ segments have equal statistical length (i.e., $a=a_{\mathrm{A}}=a_{\mathrm{B}}$ ). For the general case of $a_{\mathrm{A}} \neq a_{\mathrm{B}}$, the interfacial profile deviates from the sigmodial shape [38], and thus one needs to solve the Euler-Lagrange equation for $\phi(z)$.
The focus of our current study has been limited to the effects of chain discreteness and the width of the surface profile. Of course, there will be other effects that violate the Silberberg argument [1], thus enhancing the entropic surface segregation. An obvious couple are chain stiffness and excluded-volume interactions $[39,40]$. We plan to investigate the first of these by extending the SCFT to the worm-like chain model $[31,41,42]$ and the second by performing Monte Carlo simulations.

\section{Summary}

A numerical self-consistent field theory (SCFT) has been developed to study the entropic segregation of short polymers to the surface of a polydisperse melt. The polymers are represented by a bead-spring model consisting of monomers connected by freely-jointed bonds of arbitrary potential, and the self-consistent field is adjusted so as to produce a specified surface profile, $\phi(z)$. The resulting offlattice SCFT was applied to the special case of bidisperse melts, consisting of short and long polymers of polymerizations $N_{s}$ and $N_{l}$ and bulk volume fractions $\bar{\phi}_{s}$ and $\bar{\phi}_{l}$, respectively. For the bonded potential, we chose simple harmonic springs of statistical length $a$, and for the surface profile, we selected a sigmoidal shape with an adjustable width $\xi$. The SCFT was used to calculate the excess concentration of the short polymer, $\delta \phi_{s}(z) \equiv \phi_{s}(z)-\bar{\phi}_{s} \phi(z)$, the integrated excess, $\theta_{s} \equiv \rho_{0} \int \delta \phi_{s}(z) \mathrm{d} z$, and the entropic effect on the surface tension, $\gamma_{\mathrm{en}}$, as functions of $\xi, \alpha \equiv N_{l} / N_{s}, \bar{\phi}_{s}$, and $N_{s}$.

The SCFT calculations for $\delta \phi_{s}(z), \theta_{s}$, and $\gamma_{\mathrm{en}}$ were compared with the semi-analytical approximations in eqs. (35), (40), and (41), respectively. The expression for $\gamma_{\mathrm{en}}$ proves to be remarkably accurate, but the ones for $\delta \phi_{s}(z)$ and $\theta_{s}$ become somewhat inaccurate as $\xi$ increases. Nevertheless, the accuracy does improve with increasing polymerizations. More importantly, the semi-analytical theory reveals universal behavior that is independent of molecular details. The molecular details do, however, affect the amplitude of the segregation effects, specifically the coefficients $A$ and $\Gamma_{\infty}$ defined in eqs. (19) and (34), respectively. In general, the coefficients require a numerical calculation of the partition function, $G_{\infty}(z)$, for an infinite chain with one end fixed at $z$. However, in normal circumstances where $\xi \gtrsim a$, the coefficients are well approximated by the analytical expressions in eqs. (44) and (45). In this case, the equilibrium surface profile can be determined by a simple functional minimization of the total surface tension, $\gamma=U[\phi] / \mathcal{A}+\gamma_{\text {en }}$, where $U[\phi]$ is an energy functional for the non-bonded interactions. This fact was illustrated by deriving an improved expression, eq. (48), for the interfacial tension between immiscible polydisperse homopolymers.

As mentioned in the "Introduction", the surface segregation results from violations to the Silberberg treatment of a polymer surface. This is the underlying reason why a surface profile of finite width and discrete polymer chains cause an enrichment of short polymers at the surface. In this study, we have demonstrated that the former effect 
provides the dominant contribution once $\xi \gtrsim a$. Naturally, there will be other effects that violate the Silberberg argument, including chain stiffness and excluded-volume interactions, neither of which are accounted for by our mean-field treatment of freely-jointed chains. It will be interesting to see how large these effects are relative to the ones studied here.

We acknowledge Jamie Forrest for stimulating discussions that helped motivate this study. This work was supported by the Natural Science and Engineering Research Council (NSERC) of Canada.

\section{Author contribution statement}

PM performed the calculations under the supervision of MWM. The manuscript was written by MWM, starting from an initial draft prepared by PM.

Open Access This is an open access article distributed under the terms of the Creative Commons Attribution License (http://creativecommons.org/licenses/by/4.0), which permits unrestricted use, distribution, and reproduction in any medium, provided the original work is properly cited.

\section{References}

1. A. Silberberg, J. Colloid Interface Sci. 90, 86 (1982).

2. S.K. Kumar, M. Vacatello, D.Y. Yoon, J. Chem. Phys. 89, 5206 (1988).

3. I. Bitsanis, G. Hadziioannou, J. Chem. Phys. 92, 3827 (1990).

4. S.K. Kumar, M. Vacatello, D.Y. Yoon, Macromolecules 23 , 2189 (1990)

5. A. Yethiraj, C.K. Hall, Macromolecules 23, 1865 (1990).

6. R.S. Pai-Panandike, J.R. Dorgan, T. Pakula, Macromolecules 30, 6348 (1997).

7. K.C. Daoulas, V.A. Harmandaris, V.G. Mavrantzas, Macromolecules 38, 5780 (2005).

8. M. Müller, B. Steinmüller, K.C. Daoulas, A. RamírezHernández, J.J. de Pablo, Phys. Chem. Chem. Phys. 13 10491 (2011).

9. S.H. Anastasiadis, I. Gancarz, J.T. Koberstein, Macromolecules 21, 2980 (1988).

10. C. Jalbert, J.T. Koberstein, I. Yilgor, P. Gallagher, V. Krukonis, Macromolecules 26, 3069 (1993).

11. B.B. Sauer, G.T. Dee, J. Colloid Interface Sci. 162, 25 (1994).

12. D.T. Wu, G.H. Fredrickson, J.-P. Carton, A. Ajdari, L. Leiber, J. Polym. Sci., Part B 33, 2373 (1995).
13. M.W. Matsen, P. Mahmoudi, Eur. Phys. J. E 37, 78 (2014).

14. M.W. Matsen, J.U. Kim, A.E. Lightman, Eur. Phys. J. E 29, 107 (2009).

15. G.T. Dee, B.B Sauer, J. Appl. Polym. Sci. 134, 5 (2017).

16. Z. Qian, V.S. Minnikanti, B.B. Sauer, G.T. Dee, W.G. Kampert, L.A. Archer, J. Polym. Sci., Part B 47, 1666 (2009).

17. S.M. Sabzevari, J.D. McGraw, P. Wood-Adams, RSC Adv. 6, 91163 (2016).

18. M. Ebrahimi, M. Ansari, Y.W. Inn, S.G. Hatzikiriakos, Phys. Fluids 28, 093101 (2016).

19. A.M. Mayes, Macromolecules 27, 3114 (1994).

20. K. Tanaka, K. Tisato, T. Atsushi, T. Seiji, Macromolecules 35, 4702 (2002).

21. R. Stark, M. Kappl, H.-J. Butt, Macromolecules 40, 4088 (2007).

22. D.T. Wu, G.H. Fredrickson, Macromolecules 29, 7919 (1996).

23. D.G. Walton, A.M. Mayes, Phys. Rev. E 54, 2811 (1996).

24. V.S. Minnikanti, L.A. Archer, J. Chem. Phys. 122, 084904 (2005).

25. J.S. Lee, N.-H. Lee, S. Peri, M.D. Foster, C.F. Majkrzak, R. Hu, D.T. Wu, Phys. Rev. Lett. 113, 225702 (2014).

26. C.Y. Teng, Y.J. Sheng, H.K. Tsao, Soft Matter 12, 4603 (2016).

27. A. Hariharan, S.K. Kumar, T.P. Russell, Macromolecules 23, 3584 (1990).

28. J. van der Gucht, N.A.M. Besseling, G.J. Fleer, Macromolecules 35, 6732 (2002).

29. V.S. Minnikanti, Z. Qian, L.A. Archer, J. Chem. Phys. 126, 144905 (2007).

30. V.S. Minnikanti, L.A. Archer, Macromolecules 39, 7718 (2006).

31. M.W. Matsen, in Soft Matter, Vol. 1, Polymer Melts and Mixtures, edited by G. Gompper, M. Schick (Wiley-VCH, Weinheim, 2006).

32. D.G. Anderson, J. Assoc. Comput. Mach. 12, 547 (1965).

33. P. Mahmoudi, M.W. Matsen, Eur. Phys. J. E 39, 78 (2016).

34. S.K. Nath, J.D. McCoy, J.P. Donley, J.G. Curro, J. Chem. Phys. 103, 1635 (1995).

35. F. Schmid, J. Chem. Phys. 104, 9191 (1996).

36. A.N. Semenov, J. Phys. II 6, 1759 (1996).

37. E. Helfand, Y. Tagami, J. Chem. Phys. 56, 3592 (1972).

38. E. Helfand, A.M. Sapse, J. Chem. Phys. 62, 1327 (1975).

39. M. Müller, J. Chem. Phys. 116, 9930 (2002).

40. A. Cavallo, M. Müller, J.P. Wittmer, A. Johner, K. Binder, J. Phys.: Condens. Matter 17, S1697 (2005).

41. N. Saito, K. Takahashi, Y. Yunoki, J. Phys. Soc. Jpn. 22, 219 (1967).

42. D.C. Morse, G.H. Fredrickson, Phys. Rev. Lett. 73, 3235 (1994). 